\title{
Simulation of an aircraft radial engine misfire detection
}

\author{
Łukasz Grabowski ${ }^{1}$, Paweł Karpiński ${ }^{1, *}$, and Konrad Pietrykowski ${ }^{1}$ \\ ${ }^{1}$ Lublin University of Technology, Faculty of Mechanical Engineering, Department of Thermodynamics, Fluid Mechanics and Aviation \\ Propulsion Systems, Nadbystrzycka 36 Str., 20-618 Lublin, Poland
}

\begin{abstract}
The misfire phenomenon is particularly unfavourable in aircraft engines because it affects the stability and reliability of work. This paper presents the algorithm for detecting ignition failure in a radial aircraft engine. The Crankshaft Velocity Fluctuation method was applied, which consists in analysing changes in the crankshaft speed signal as a function of time. A zero-dimensional model of the aircraft engine was developed in order to perform the research. The validation of the model was performed using the results from the test bench. The model was subjected to simulation tests in fixed operating conditions. Based on the engine speed signal obtained as a result of the simulation, the normalized second derivative of the signal was determined based on the adopted algorithm. On the basis of this derivative, a criterion was defined to assess the occurrence of the misfire phenomenon. The results of the calculations can be compared in future with the results of the real engine tests.
\end{abstract}

\section{Introduction}

The phenomenon of misfiring in piston engines has a negative effect on the emission of toxic substances. It also results in deterioration of the catalyst or may lead to its damage. In addition, it negatively affects the stability of the engine - there is a change in the distribution of forces acting on subsequent cranks. Misfire phenomenon causes variability and uniqueness of a combustion process. This can be seen by analysing measured cylinder pressure from radial engine with multifractal approach and illustrate this phenomenon by means of wavelet results like in the work [1]. Ignition losses in SI engines can be caused by various causes: failure of ignition coils, failure or wear of spark plugs, failure of the electronic ignition control module, malfunction of the fuel system. Also changing fuel from liquid state into gas state also can cause disturbances in air fuel mixture and affect in misfire phenomenon both in SI and CI engines. Gęca and Litak investigated fluctuations during the combustion of hydrogen-enriched gasoline an IC-SI engine in the paper [2]. The authors of the work [3] by applying the statistical and multifractal methods to the corresponding time pressure series showed the considerable changes in engine dynamics for a different equivalence ratio decreases from 0.781 to very lean conditions.

The other causes of misfiring in CI engines are different due to the self-ignition process. In the study [4] the misfire in the locomotive diesel engine was investigated using non-linear analysis based on the deterministic chaos theory. For the analysis, vibroacoustic signals obtained from an exhaust locomotive engine were used. A separate group of methods for analysing the phenomenon of misfire are methods based on neural networks. In work [5], based on the general work parameters of the supercharged diesel engine, the artificial neural network model (ANN) was designed to detect the misfire. A similar model of ANN was developed in paper [6], where investigated the phenomenon of misfire in an ethanol-powered HCCI engine. The experimental engine test results were used as input data to the model. Examples of other works in which neural networks are used to detect the misfire are papers [7-9].

One of the basic methods for detecting misfire is the Crankshaft Velocity Fluctuation (CVF) method. It involves the use of a crankshaft speed sensor to analyse engine speed fluctuations. The fluctuation is caused by the lack of combustion pressure in a given cylinder, and therefore the lack of torque generated in a given part of the crank-piston system. This type of method was used in work [10], which was verified by performing test runs on the track for various loads, gear ratios and accelerations of the vehicle.

In car engines, On-board Diagnostics System (OBDII) controls the misfiring. In the work [11], various methods of misfire detection in accordance with the OBD-II system are presented. However, this standard does not apply to aircraft engines. The detection of the misfire phenomenon in the case of aircraft engines is particularly important from the point of view of obtaining stable and reliable operation of the engine.

Paper [12] presents a method meeting the OBD-II requirements for misfire detection based on a rotational acceleration analysis. In the algorithm developed by the authors, the "engine roughness" parameter was calculated based on the momentary engine speed and engine load. In addition, error correction functions were applied during fast-changing operating conditions.

\footnotetext{
* Corresponding author: pawel.karpinski@pollub.edu.pl
} 
Paper [13] presents the concept of a system that allows misfire detection in SI engines based on the analysis of the crankshaft speed change. The tests were carried out on the basis of a 12-cylinder engine.

In addition, attempts have been made with other methods to detect misfiring. In the study [14] a method based on Ionization Current Monitoring was presented.

It is possible to use digital filtering to detect misfiring. An example of this is the research presented in work [15], where the misfire phenomenon in a highspeed four-cylinder engine was analysed using this method. In work [16], a model of ignition detection using Kalman filter was used.

The authors of the work [17] used in their work the Fourier transform to analyse the pressure pulsation of the exhaust gases in order to detect the misfire of the 12cylinder $\mathrm{V}$-engine. The method of misfire detection on the basis of analysis in the time-frequency domain was presented in the work [18]. The presented method has been experimentally verified using a spark-ignition engine.

The work [19] presents a combination of two methods: digital filtering with statistical recognition of patterns in real time. Periodic and non-periodic ignition misfires have been characterized by separate algorithms.

Most of the work devoted to investigating the phenomenon of ignition loss focuses on the analysis of in-line or $\mathrm{V}$ engines. Therefore, there is a need to investigate this process in the case of an aircraft radial engine. The authors of the paper [20] analysed IMEP in 9-cylinder radial engine. There is misfire especially at below $1200 \mathrm{rpm}$. At $500 \mathrm{rpm}$ misfire occurs every second cycle. Results of CFD model of the aircraft radial engine presented in paper [21] show that combustion prolongs in time in the case of lack of ignition in one of two spark plugs.

One of the methods of testing internal combustion engines is to develop an engine model and perform simulation calculations. An example of such an approach is the research presented in paper [22], in which the model for determining the heat of the engine and heat flux penetrating through each of the engine's surfaces was used. The purpose of this work was to simulate the aircraft radial engine ASz-62IR model for misfire analysis. The analysis consisted in using the developed algorithm and defining the criterion for checking the occurrence of misfire based on the engine speed signal as a function of time. Section 2 presents the algorithm used to detect the misfire phenomenon. The next Section presents a zero-dimensional engine model used for research. In Section 4, the results obtained and their analysis are presented. The misfire condition for the proposed criterion was obtained.

\section{Algorithm}

The following algorithm is used to analyse the ignition failure in the cylinder. As a result of the simulation, the signal of engine speed was recorded as a function of time (1):

$$
f=\omega(t)
$$

Then the first derivative (2) was calculated, followed by the second derivative (3) of the engine speed as a function of time:

$$
\begin{gathered}
\varepsilon(t)=\omega^{\prime}(t) \\
\gamma(t)=\varepsilon^{\prime}(t)=\omega^{\prime \prime}(t)
\end{gathered}
$$

The course of the second derivative was normalized according to equations (4) and (5) presented below:

$$
\begin{gathered}
\gamma_{1}(t)=\gamma(t)+\left|\gamma_{\text {min }}\right| \\
\gamma_{n}(t)=\frac{\gamma_{1}(t)}{\gamma_{1, \max }}
\end{gathered}
$$

In the next step, the signal was divided into $k$ intervals with a length corresponding to $1 / 9$ of the average duration of one cycle $T_{\text {mean }}$. The number of intervals $k$ resulted from the duration of the measurement, which was 27 seconds. For the determined intervals, local maxima (6) for the normalized signal of engine speed were determined:

$$
\gamma_{n, \max _{i}} \quad i=1, \ldots, k
$$

Finally, the following criterion was adopted to determine the occurrence of misfiring (7):

$$
\gamma_{n, \max _{i}} \leq X \text { - misfire in cylinder for } i \text { interval }
$$

$\gamma_{n, \max _{i}}>X-$ correct ignition in cylinder for $i$ interval

The value of $X$ was determined on the basis of the analysis of the values of local maxima for 1st cylinder (Section 4). Additional analysis of a signal comprising averaging, accumulation, or statistical filtering as potential different decision errors of process statistical error compensation should be included for further research.

\section{Engine model}

The object of this part of study was an ASz-62IR piston radial engine with spark ignition and supercharging performed by a single-speed centrifugal compressor.

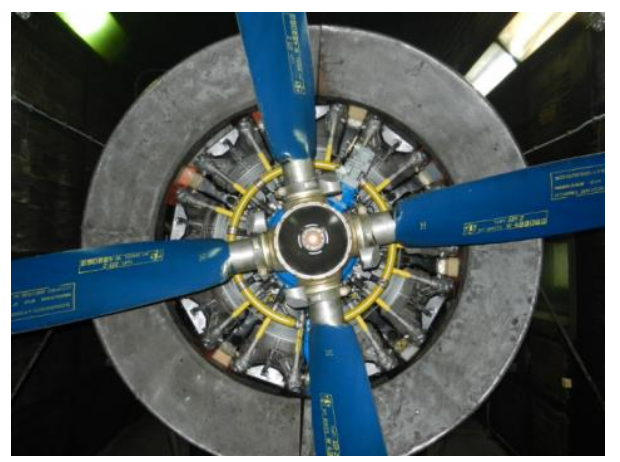

Fig. 1. Aircraft radial engine ASz-62IR - research object. 
The engine has 9 air-cooled cylinders. Thanks to the compressor, the charging pressure increases, which ensures that the nominal power is maintained up to a height of $1500 \mathrm{~m}$.

The engine is equipped with a dual ignition system, an inertia starter and an electronically controlled injection system. The planetary gear allows to reduce the rotational speed of the propeller relative to the speed of the crankshaft. The construction of the engine enables driving various types of aggregates and tools, thanks to which it is suitable for installation in aircraft used in agriculture. ASz-62IR can be mounted on Antonov An2, PZL M18 Dromader or DHC-3 Otter. The engine met the certification conditions in Poland, Canada, Brazil, China, Argentina and the USA. The basic engine parameters are presented in Tab. 1.

Table 1. Basic technical parameters of the tested engine (source: WSK PZL Kalisz, Technical description of aircraft engine ASz-62IR 16, Warsaw 1976).

\begin{tabular}{|l|l|}
\hline Engine type & $\begin{array}{l}\text { Piston engine, four-stroke, } \\
\text { spark ignited, radial, } \\
\text { supercharged }\end{array}$ \\
\hline Type of supercharging & Centrifugal compressor \\
\hline Capacity of cylinders & $29.87 \mathrm{dm}^{3}$ \\
\hline Bore & $155.5 \mathrm{~mm}$ \\
\hline Stroke for 1st cylinder & $174.5 \mathrm{~mm}$ \\
\hline Compression ratio & $6.4: 1$ \\
\hline Take-off power & $745 \mathrm{~kW}(2200 \mathrm{rpm})$ \\
\hline Firing order & $1,3,5,7,9,2,4,6,8$ \\
\hline
\end{tabular}

Based on technical parameters of the engine, a zerodimensional model was developed in the AVL BOOST program. This model was also used in the work [23], in which the emission of substances toxic to given working conditions was examined. This model is defined on the basis of the first law of thermodynamics. This means that the method of spreading the flame in the cylinder is assumed, and the time is an independent variable. The mass burning rate is based on literature or experimental data $[24,25]$.

The combustion model described in the Vibe function was adopted, which is determined by the beginning and duration of combustion, the shape parameter $\mathrm{m}$ and the parameter $a$. The Vibe function has the form (8):

$$
\begin{gathered}
\frac{d x}{d \alpha}=\frac{\alpha}{\Delta \alpha_{c}} \cdot(m+1) \cdot y^{m} \cdot e^{-a \cdot y^{m+1}} \\
d x=\frac{d Q}{Q} \\
y=\frac{\alpha-\alpha_{0}}{\Delta \alpha_{c}}
\end{gathered}
$$

Where:

$Q$ - supplied heat,

$\alpha$ - crank angle,

$\alpha_{0}-$ start of combustion expressed in crank angle,
$\Delta \alpha_{c}$ - duration of combustion expressed in crank angle, $m$ - shape parameter,

$a-$ Vibe parameter, for perfect combustion is equal to 6.9. [26]

By integrating the Vibe function after the crank angle, a mass fraction burned (11) can be calculated:

$$
x \int \frac{d x}{d \alpha} d \alpha=1-e^{-a \cdot y^{m+1}}
$$

Where:

$x$ - mass fraction burned, MFB. [26]

Parameters of the combustion model were determined based on experimental data loaded into the Burn module. This module allows to determine the parameters of the combustion model based on the actual operating characteristics of the tested engine. On the basis of the measured pressure signal read into the program, the parameters of the combustion model and the rate of heat release (ROHR) as a function of crank angle were determined. The indicated pressure was measured on the engine test bench belonging to the company WSK PZL Kalisz. OPTRAND optical pressure sensor installed in the central part of the cylinder head was used for the measurement. The signal from measuring sensor was recorded with a sampling frequency of $100 \mathrm{kHz}$ using National Instruments measuring equipment and LabView software. The registered cylinder pressure signal (Fig. 2) has been loaded into the Pressure Trace tab. [27]

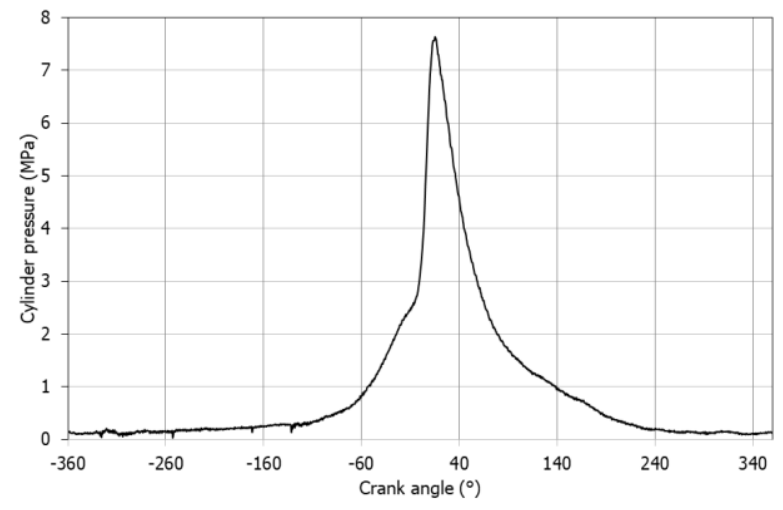

Fig. 2. Measured cylinder pressure [27].

In the settings of the Burn module, a two-zone combustion model and a classic model of species transport were chosen. The operation point has been defined for an engine speed of $2200 \mathrm{rpm}$. The model of Woschni 1978 was adopted for the heat exchange model. Detailed parameters adopted in the Burn module are presented in Fig. 3. [24, 27] 


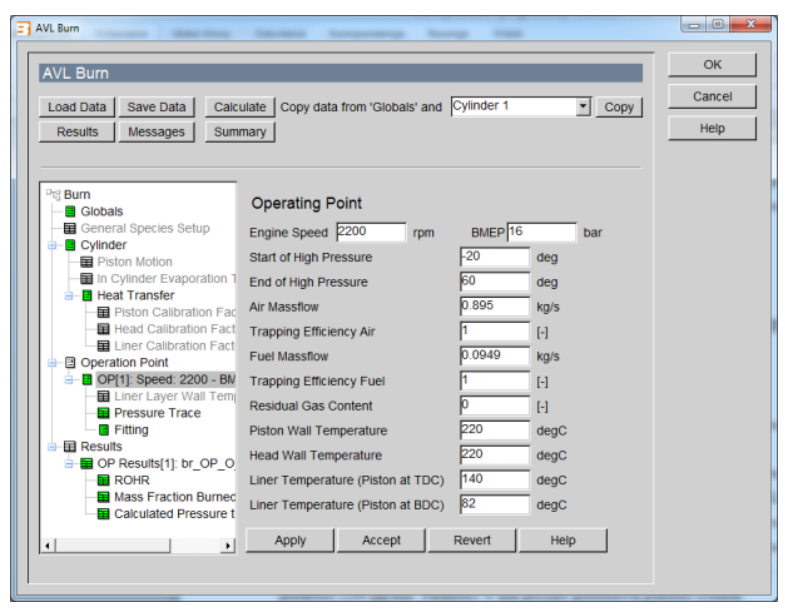

Fig. 3. Parameters set in the Burn module [27].

As a result of calculations, the following parameters of the combustion model were determined: start of combustion $S o C=-4.74^{\circ}$, combustion duration $C D=$ $28.4^{\circ}$, shape parameter $m=1.32$. Based on these parameters and the Vibe function equation (8), the rate of heat release (ROHR) was determined as a function of the crank angle (Fig. 4). Although the curve determined on the basis of experimental tests does not ideally coincide with the curve determined on the basis of the Vibe function, the amount of heat released in one cycle is preserved. [27]

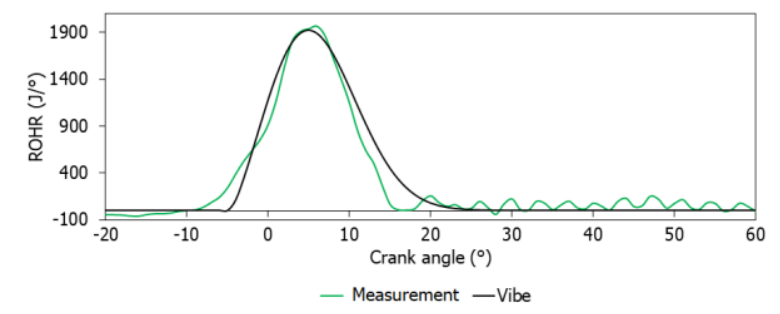

Fig. 4. Rate of heat release as a function of crank angle [27].

As a model of heat exchange, the Woschni model (1978) was adopted, which is a complex function of a number of parameters, including cylinder volume, cylinder temperature and pressure [28].

Heat exchange also takes place around the inlet and outlet valves. Therefore, a modified Zapf model was used, which takes into account heat exchange and temperature coefficients. The program defined the surface of the piston, head and temperature of these elements.

In modeling the air and fuel flow through pipes to the engine cylinders, only the diameters and lengths of the pipes connecting individual engine elements are taken into account. The timing phases have been determined based on the valve lift characteristics given by the engine manufacturer. The size of valve seats was also introduced and flow resistances were defined.

Fig. 5 shows the ASz-62IR engine model created in the AVL BOOST program.

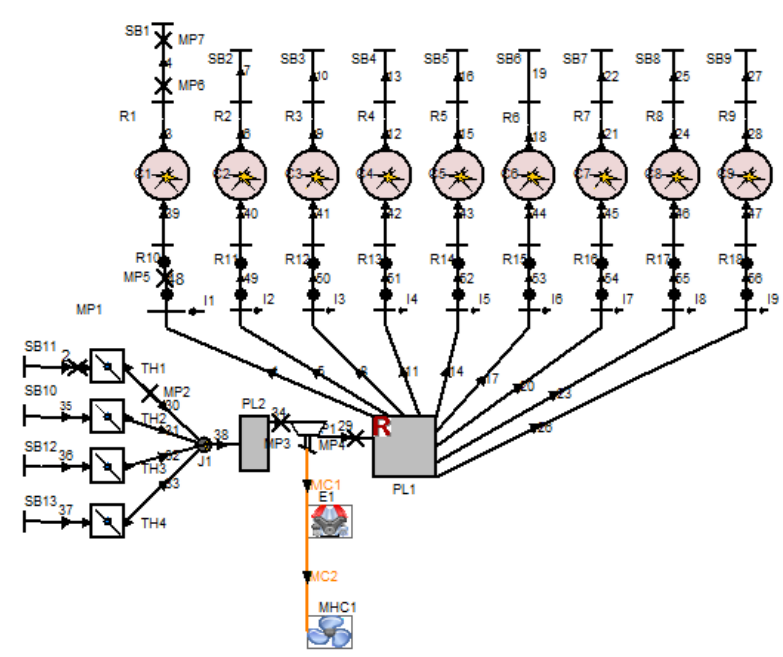

Fig. 5. The engine test model created in the AVL BOOST program.

The air flow in the developed model is realized through the intake pipes, the group of throttles, and then the centrifugal compressor. The compressor is driven by the transmission from the crankshaft. In the model this has been accomplished by using a mechanical connection MC1 with the engine block. Large volumes of small length in the intake duct have been replaced with Plenum type elements. The injection system is a set of 9 injectors located in the intake ducts. The mass flow of injectors varies dynamically depending on the mass flow of air and is adapted to the assumed composition of the fuel-air mixture. In selected places, measuring points and flow restriction elements were added on the connecting pipes. The elements used to create the model are presented in Table 2 [27].

Table 2. Components of the engine model.

\begin{tabular}{|c|c|c|}
\hline Element & Name & Symbol \\
\hline & System Boundary & $\mathrm{SB}$ \\
\hline & Pipe & number \\
\hline & Junction & $\mathrm{TH}$ \\
\hline & Plenum & $\mathrm{J}$ \\
\hline
\end{tabular}

It was assumed that the engine is powered by gasoline with a calorific value of $43.5 \mathrm{MJ} / \mathrm{kg}$ and an air- 
fuel ratio of $14.5 \mathrm{~kg}$ of air $/ \mathrm{kg}$ of fuel. It was assumed that the inlet air has a temperature of $24.85^{\circ} \mathrm{C}$ and a pressure of 1 bar. It was assumed that the engine works with an excess air ratio $\lambda$ equal to 0.65 . The chemical transport model in the working medium was defined as Classic Species Transport. The throttle operation has been defined based on data from the engine manufacturer. The firing order was specified in element E1. A compressor map from the manufacturer was loaded into the model. It was assumed that burned and unburned products have identical pressure and temperature, but different composition.

The model has been extended by the ability to perform calculations in dynamic states with a loaded engine. The power receiver was a propeller $(\mathrm{MHC1}$ in Fig. 5) mechanically connected to the engine (reference MC2 in Fig. 5). Due to the fact that simulation studies relate to dynamic states, mass moments of inertia of elements have been defined. For the engine, the value of the polar moment of inertia being the equivalent torque of all rotating masses reduced to the axis of the crankshaft has been introduced. A mass moment of inertia of $69 \mathrm{~kg} \cdot \mathrm{m} 2$ was introduced in the Mechanical Consumer model element. It was assumed that the load on the propeller is $5400 \mathrm{Nm}$.

The aim of the research was to analyse the momentary engine speed change due to the lack of ignition in one cylinder. Burning in one cylinder has been intentionally switched off.

In order to perform the analysis of engine speed changes, calculations were made to determine the engine's operating conditions. The difference in the engine speed in subsequent cycles below $0.5 \%$ was assumed for determining the working conditions. These operating conditions were obtained after approximately 70 calculation cycles of the engine operating cycle.

The calculations were performed for the case of the take-off power at the engine speed of $2200 \mathrm{rpm}$ and the pressure in the intake manifold equal to $140 \mathrm{kPa}$. Ignition in 1 cylinder was switched off.

\section{Results and discussion}

Fig. 6 shows the signal of the engine speed as a function of time obtained as a result of the simulation calculations.

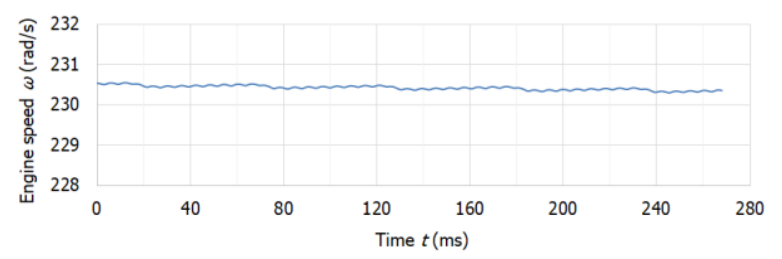

Fig. 6. Engine speed as a function of time.

The beginning of the signal coincides with the moment of ignition in the 4th cylinder. It is possible to observe temporary drops in rotational speed. This means that the ignition in one of the cylinders does not occur. After the temporary drop in speed, its value increases slightly. Local maxima correspond to the ignitions occurring in subsequent cylinders. The decreasing speed trend results from the fact that its value is still determined in subsequent calculation cycles.

Fig. 7 and Fig. 8 show respectively the first and second derivative of the engine speed as a function of time. On both signals you can observe a number of maxima corresponding to the work of the next cylinders. The maxima value remains constant, while in selected cases it drops significantly. The drop in value is cyclical, which means that the ignition in one of the cylinders does not occur.

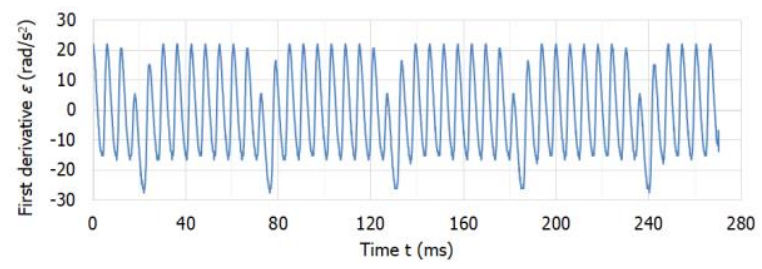

Fig. 7. The first derivative of engine speed as a function of time.

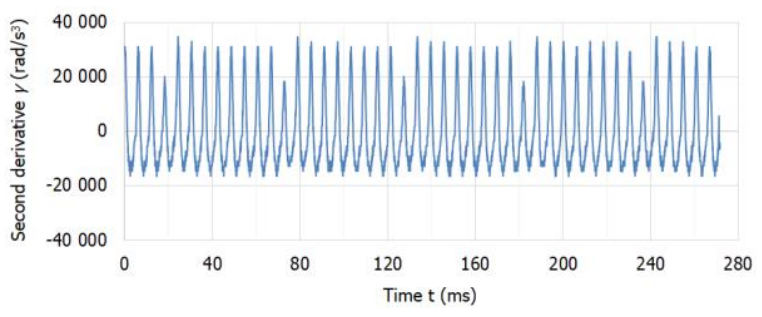

Fig. 8. The second derivative of engine speed as a function of time.

Based on the second derivative of the engine speed, the signal was normalized (Fig. 9), and then the misfire detection criterion was applied in accordance with the algorithm presented in the Section 2 of this paper.

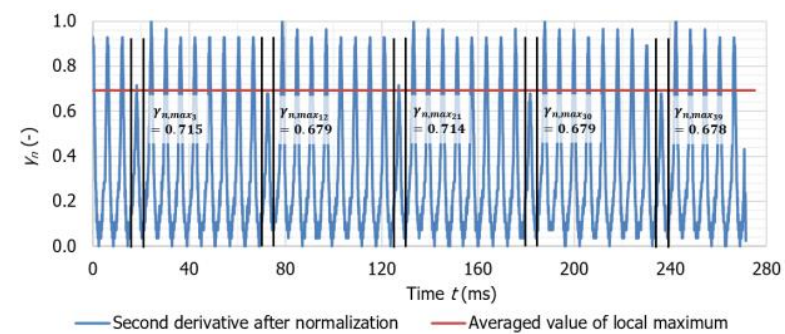

Fig. 9. The second derivative of engine speed as a function of time.

By comparing the time intervals with the firing sequence, it is possible to determine which cylinder corresponds to the specified maximum. In this way, the maxima and interval numbers to which 1 st cylinder corresponds are determined. These data are presented in Tab. 3. 
Table 3. Maxima corresponding to 1 st cylinder.

\begin{tabular}{|c|c|c|}
\hline $\begin{array}{c}\text { Number of } \\
\text { interval }\end{array}$ & Time interval & $\begin{array}{c}\text { Value of local } \\
\text { maximum }\end{array}$ \\
\hline 3 & $15.14-21.20 \mathrm{~ms}$ & 0.715 \\
\hline 12 & $75.72-81.78 \mathrm{~ms}$ & 0.679 \\
\hline 21 & $124.19-130.25 \mathrm{~ms}$ & 0.714 \\
\hline 30 & $178.73-184.79 \mathrm{~ms}$ & 0.679 \\
\hline 39 & $233.28-239.34 \mathrm{~ms}$ & 0.678 \\
\hline
\end{tabular}

On the basis of the maxima values, the threshold $X=0.715$ for the criterion (7) presented in the algorithm in Section 2 is determined. This means that for maxima with the value equal to or less than 0.715 occur the misfire.

\section{Conclusions}

On the basis of the analysis of changes in rotational speed and its derivatives as a function of time, it is possible to evaluate the operation of individual cylinders in the absence of ignitions.

As a result of the simulation tests of the developed model of the radial engine, the signal of the rotational speed of the engine as a function of time was obtained. As a result of switching off the ignition, there was a cyclic rotational speed disturbance in 1 cylinder. It was caused by the lack of torque generated due to the rapid increase in pressure in a given cylinder. Based on the obtained signal, a developed algorithm was used to detect the misfire. The criterion introduced in the algorithm was determined after performing calculations and analysis (calculation of the second derivative of the signal and normalization of the speed signal).

During engine operation in real conditions external factors disturbing the engine speed in variable operating conditions may occur. Therefore, in further research, it will be necessary to verify the algorithm adopted based on bench tests and apply the necessary corrections to eliminate possible errors.

\section{References}

1. M. Gęca, M. Wendeker, J. Vibroeng. 14(2), 582- 591 (2012)

2. M. Gęca, G. Litak, Measurement 108, 18-25 (2017)

3. G. Litak, M. Gęca, B. F. Yao, G. X. Li, Z. fuer Naturforsch. 64a, 393-398 (2009)

4. P. Boguś, J. Merkisz, Mech. Syst. Signal Process. 19(4), 881-899 (2005)

5. B. Liu, C. Zhao, F. Zhang, T. Cui, J. Su, Appl. Therm. Eng. 55(1-2), 26-32 (2013)

6. B. Bahri, A. A. Aziz, M. Shahbakhti, M. F. M. Said, Appl. Energy 108, 24-33 (2013)

7. Z. J. Wu, A. Lee, SAE Technical Paper No. 980515 (1998)
8. W. B. Ribbens, J. Park, D. Kim, ICASSP-94, 1994 IEEE International Conference on Vol. 2, pp. II-593 (1994)

9. H. Nareid, N. Lightowler, SAE Technical Paper No. 2004-01-1363 (2004)

10. D. Moro, P. Azzoni, G. Minelli, SAE Technical Paper No. 980521 (1998)

11. J. Merkisz, P. Boguś, R. Grzeszczyk, Journal of Kones 8(1-2), 326-341 (2001)

12. M. Klenk, W. Moser, W. Mueller, W. Wimmer, SAE Technical Paper No. 930399 (1993)

13. J. Förster, A. Lohmann, M. Mezger, K. RiesMüller, SAE Technical Paper No. 970855 (1997)

14. A. Lee, J. S. Pyko, SAE Technical Paper No. 950003 (1995)

15. S. Kuroda, Y. Shimasaki, H. Igarashi, S. Maruyama, T. Asaka, JSAE Review 16(4), 387390 (1995)

16. U. Kiencke, Control Eng. Pract. 7(2), 203-208 (1999)

17. M. Ceccarani, C. Rebottini, R. Bettini, SAE Technical Paper No. 980518 (1998)

18. N. Cavina, E. Corti, G. Minelli, G. Serra, SAE Technical Paper No. 2002-01-0480 (2002)

19. A. W. Osburn, T. M. Kostek, M. A. Franchek, Mech. Syst. Signal Process. 20(8), 2232-2258 (2006)

20. J. Czarnigowski, M. Wendeker, P. Jakliński, K. Pietrykowski, A. Nazarewicz, M. Gęca, T. Zyska, SAE Technical Paper No. 2007-01-2074 (2007)

21. K. Pietrykowski, SAE Technical Paper No. 201401-2578 (2014)

22. Ł. Grabowski, Z. Czyż, K. Kruszczyński, SAE International No. 2014-01-2883 (2014)

23. Z. Czyż, Ł. Grabowski, K. Pietrykowski, J. Czarnigowski, M. Porzak, Measurement 113, 46-52 (2018)

24. T. Tulwin, Procedia Eng. 157, 255-263 (2016)

25. T. Tulwin, R. Sochaczewski, ITM Web of Conferences 15, 07016 (2017)

26. AVL BOOST Theory 11/2013.

27. P. Karpiński, Ł. Grabowski, Transactions of the Institute of Aviation 3(244), 305-317 (2016)

28. K. Pietrykowski, T. Tulwin, SAE International Journal of Engines 8(1), 82-88 (2015) 